\title{
Lower bounds on the projective heights of algebraic points
}

\author{
by \\ Charles L. Samuels (Austin, TX)
}

1. Introduction. Let $K$ be any number field and $v$ a place of $K$ extending the place $p$ of $\mathbb{Q}$. Let $K_{v}$ denote the completion of $K$ at $v$ and $\mathbb{Q}_{p}$ the completion of $\mathbb{Q}$ at $p$. Write $D_{v}=\left[K_{v}: \mathbb{Q}_{p}\right]$ and $D=[K: \mathbb{Q}]$ for the local and global degrees. Let $\|\cdot\|_{v}$ be the unique absolute value on $K_{v}$ that extends the $p$-adic absolute value on $\mathbb{Q}_{p}$ and define $|\cdot|_{v}=\|\cdot\|_{v}^{D_{v} / D}$. We note that $|\cdot|_{v}$ satisfies the product formula

$$
\prod_{v}|\alpha|_{v}=1
$$

for all $\alpha \in K^{\times}$where the product is taken over all places $v$ of $K$.

Define the projective height of a point $\mathbf{x}=\left(x_{0}, \ldots, x_{n}\right) \in \mathbb{P}^{n}(K)$ by

$$
\log H(\mathbf{x})=\sum_{v} \log \max _{i}\left|x_{i}\right|_{v}
$$

and note that by the product formula $H$ is well defined on $\mathbb{P}^{n}(K)$. By our choice of absolute values, the definition of $H$ does not depend on $K$ and therefore defines a function on $\mathbb{P}^{n}(\overline{\mathbb{Q}})$. We define the Weil height $h(\alpha)$ of a point $\alpha \in \overline{\mathbb{Q}}$ by

$$
h(\alpha)=H((1, \alpha)) .
$$

By Kronecker's theorem, $\log h(\alpha) \geq 0$ with equality if and only if $\alpha$ is 0 or a root of unity. In 1933, Lehmer [3] asked whether there exists a constant $\varrho>1$ such that

$$
\operatorname{deg}(\alpha) \log h(\alpha) \geq \log \varrho
$$

in all other cases. In particular, he asked whether we may take $\varrho$ to be the larger real root of $x^{10}+x^{9}-x^{7}-x^{6}-x^{5}-x^{4}-x^{3}+x+1$.

Lehmer's problem is still open today though an affirmative answer has been given for certain classes of algebraic numbers. Smyth [5] proved that

2000 Mathematics Subject Classification: Primary 11R04.

Key words and phrases: projective height, Weil height, Lehmer's problem. 
if $\alpha \neq 0$ and the minimal polynomial of $\alpha$ is not reciprocal then (1.1) holds with $\varrho$ the real root of $x^{3}-x-1$. It is a consequence of a theorem of Schinzel [4] that if $\alpha$ is totally real we may take $\log \varrho=\frac{1}{2} \log ((1+\sqrt{5}) / 2)$. If we further assume that $\alpha$ is an algebraic integer then the same bound holds without $\operatorname{deg}(\alpha)$ appearing on the left hand side of (1.1). In this case, Schinzel's lower bound is best possible by taking $\alpha=(1+\sqrt{5}) / 2$. The best unconditional result toward answering Lehmer's problem is a theorem of Dobrowolski [2] which gives a lower bound on $\operatorname{deg}(\alpha) \log h(\alpha)$ which tends to 0 slowly as $\operatorname{deg}(\alpha) \rightarrow \infty$.

In a slightly different direction, Zhang [7] showed that there exists $\varrho>1$ such that

$$
\log h(\alpha)+\log h(1-\alpha) \geq \log \varrho
$$

whenever $\alpha$ is not 0,1 or a primitive 6 th root of unity. Zagier [6] used elementary methods to show that (1.2) holds with $\log \varrho=\frac{1}{2} \log ((1+\sqrt{5}) / 2)$ with cases of equality identified. As Zagier notes, it is interesting that this is the same lower bound that appears in Schinzel's bound [4] on the height of a totally real algebraic integer. Our goal is to show that the results of Schinzel and Zagier are in fact consequences of a more general theorem.

Our proof will apply the methods of Beukers and Zagier [1] who generalized the results of [6] in the following way. Let $\alpha_{1}, \ldots, \alpha_{r}$ be non-zero algebraic numbers such that $\alpha_{1}+\cdots+\alpha_{r}=N$ and $\alpha_{1}^{-1}+\cdots+\alpha_{r}^{-1} \neq N$ for some integer $N$. Then

$$
\sum_{i=1}^{r} \log h\left(\alpha_{i}\right) \geq \frac{1}{2} \log \frac{1+\sqrt{5}}{2}
$$

with cases of equality. We will further generalize this theorem so that $N$ may be any totally real algebraic integer. Then by taking $r=1$ we are able to recover Schinzel's result.

2. Main results. Suppose that $r, n_{1}, \ldots, n_{r}$ are positive integers and $K$ is a field. Then we write $\mathcal{P}(K)=\mathbb{P}^{n_{1}}(K) \times \cdots \times \mathbb{P}^{n_{r}}(K)$ and denote the coordinates by $\mathbf{x}=\left(\mathbf{x}_{0}, \ldots, \mathbf{x}_{r}\right)$ with $\mathbf{x}_{i}=\left(x_{i 0}, \ldots, x_{i n_{i}}\right)$. If $\mathbf{x}$ has $x_{i j} \neq 0$ for all $i, j$ let $\mathbf{x}^{-1}$ be the point obtained by replacing each coordinate $x_{i j}$ of $\mathbf{x}$ with $x_{i j}^{-1}$. Following [1], choose any subset $I$ of $\left\{i \mid n_{i}=1\right\}$ and let $E=\{(i, 0) \mid i \in I\}$. We refer to $E$ as the set of exceptional index pairs. Index pairs not in $E$ are called regular index pairs. If a regular index pair appears in a monomial of a polynomial $Q(\mathbf{x})$, then we say the monomial is a regular monomial of $Q$. Otherwise, the monomial is called an exceptional monomial. Also write $\|Q\|_{v}$ to denote the sum of the $v$-adic absolute values (using $\|\cdot\|_{v}$ ) of the coefficients of $Q$. 
Let $F$ be a multihomogeneous polynomial over $\overline{\mathbb{Q}}$ of multidegrees $d_{1}, \ldots, d_{r}$ so that $F$ defines a zero set in $\mathcal{P}(\overline{\mathbb{Q}})$. The degree of $F$ in the variable $x_{i j}$ is denoted $d_{i j}$ and define $\widetilde{d}_{i}=-d_{i}+\sum_{j} d_{i j}$. Then we set

$$
\delta=\max \left\{\max _{i \in I}\left\{\frac{\widetilde{d}_{i}+d_{i 1}}{n_{i}+1}\right\}, \max _{i \notin I}\left\{\frac{\widetilde{d}_{i}}{n_{i}+1}\right\}\right\}
$$

and assume that $F$ has the following properties:

(i) the coefficients of $F$ are totally real algebraic integers,

(ii) the coefficients of regular monomials of $F$ are integers.

Then for $v$ Archimedean define

$$
c(F, v, i, j)=\left\|\frac{\partial F}{\partial x_{i j}}\right\|_{v} .
$$

In [1], Beukers and Zagier consider only polynomials $F$ having integer coefficients, so clearly $c(F, v, i, j)$ does not depend on the place $v$. In fact, $c(F, v, i, j)$ is defined in [1] using the usual absolute value on the complex numbers rather than $\|\cdot\|_{v}$. Since we assume only the weaker conditions (i) and (ii), $c(F, v, i, j)$ may indeed depend on $v$ as the notation suggests. Therefore, we require the absolute value $\|\cdot\|_{v}$ in this definition.

However, in the special case that $(i, j) \notin E, c(F, v, i, j)$ depends only on the regular monomials of $F$. So by property (ii), $c(F, v, i, j)$ depends only on the monomials of $F$ having integer coefficients, and therefore, does not depend on $v$. Then we may define

$$
C_{F}=C_{F}(E)=\max _{(i, j) \notin E} c(F, v, i, j)
$$

and by our remarks above, $C_{F}$ does not depend on $v$. We now state our main theorem, which is a direct generalization of the main theorem in [1].

THEOREM 2.1. Let $F$ be a multihomogeneous polynomial with properties (i) and (ii) above for some exceptional set $E$. If $\mathbf{x} \in \mathcal{P}(\overline{\mathbb{Q}})$ is such that $F(\mathbf{x})=0, \prod_{i, j} x_{i j} \neq 0$ and $F\left(\mathbf{x}^{-1}\right) \neq 0$ then

$$
\sum_{i=1}^{r}\left(n_{i}+1\right) \log H\left(\mathbf{x}_{i}\right) \geq \log \varrho
$$

where $\varrho$ is the unique real root larger than 1 of $x^{-2}+C_{F}^{-1} x^{-\delta}=1$.

Once again, we note that our theorem generalizes [1] in that we allow the coefficients of $F$ to come from a potentially larger set. While the main theorem in [1] requires these coefficients to be integers, we allow some of them to be any totally real algebraic integers.

Before we prove Theorem 2.1 we demonstrate its relationship to our problem. Consider $r$ non-zero algebraic numbers $\alpha_{1}, \ldots, \alpha_{r}$ such that $\alpha_{1}+\cdots+\alpha_{r}=N$ and $\alpha_{1}^{-1}+\cdots+\alpha_{r}^{-1} \neq N$. Corollary 2.1 of [1] gives 
a lower bound on $\sum_{i=1}^{r} \log h\left(\alpha_{i}\right)$ when $N$ is an integer. We apply Theorem 2.1 to prove a direct generalization of this result.

Corollary 2.2. Suppose $\alpha_{1}, \ldots, \alpha_{r}$ are non-zero algebraic numbers and $N$ is a totally real algebraic integer. If $\alpha_{1}+\cdots+\alpha_{r}=N$ and $\alpha_{1}^{-1}+\cdots+\alpha_{r}^{-1}$ $\neq N$ then

$$
\sum_{i=1}^{r} \log h\left(\alpha_{i}\right) \geq \frac{1}{2} \log \frac{1+\sqrt{5}}{2}
$$

with equality when $r=1$ and $\alpha_{1}=(1+\sqrt{5}) / 2$.

Proof. Write $\alpha_{i}=\alpha_{i 1}$ for all $i$ and suppose that the $\alpha_{i 0}$ are algebraic numbers. We consider the point

$$
\alpha=\left(\alpha_{10}, \alpha_{11}\right) \times \cdots \times\left(\alpha_{r 0}, \alpha_{r 1}\right) \in\left(\mathbb{P}^{1}(\overline{\mathbb{Q}})\right)^{r} .
$$

We will apply Theorem 2.1 to this point with $I=\{1, \ldots, r\}$ so we have $E=$ $\{(1,0), \ldots,(r, 0)\}$. Let $F$ be the homogeneous version of $x_{10}+\cdots+x_{r 0}-N$. That is,

$$
F(\mathbf{x})=\sum_{i=1}^{r} x_{i 1} \prod_{j \neq i} x_{j 0}-N \prod_{j} x_{j 0}
$$

and note that $F$ has properties (i) and (ii). It is clear that $c(F, v, i, j)=1$ for all $(i, j) \notin E$ so that $C_{F}=1$. We also have $n_{i}=1, d_{i}=1$ and $d_{i 1}=1$ so that $\delta=1$. Then by Theorem 2.1,

$$
\sum_{i=1}^{r} 2 \log H\left(\alpha_{i 0}, \alpha_{i 1}\right) \geq \log \varrho
$$

where $\varrho$ is the real root larger than 1 of $x^{-2}+x^{-1}=1$. Setting $\alpha_{i 0}=1$ for all $i$ the result follows and the case of equality is clear.

Note that the case of equality in Corollary 2.2 is not unique. For example, we also have equality when $r=2, \alpha_{1}=1$ and $\alpha_{2}=(1+\sqrt{5}) / 2-1$. Several other cases of equality are given in [1] and [6] using integer values for $N$.

In the special case that $r=1$ Corollary 2.2 implies that $\log h(\alpha) \geq$ $\frac{1}{2} \log ((1+\sqrt{5}) / 2)$ for all totally real algebraic integers $\alpha \notin\{0, \pm 1\}$. Therefore, Schinzel's bound [4] on the height of a totally real algebraic integer is a corollary of our result.

COROLlary 2.3. If $\alpha$ is a totally real algebraic integer with $\alpha \notin\{ \pm 1,0\}$, then

$$
\log h(\alpha) \geq \frac{1}{2} \log \frac{1+\sqrt{5}}{2} .
$$

3. Proof of Theorem 2.1. We begin with some additional notation. Recall that for a point $\mathbf{x} \in \mathcal{P}(K)$ for some field $K$ we denote the coordinates 
$\mathbf{x}=\left(\mathbf{x}_{1}, \ldots, \mathbf{x}_{r}\right)$ with $\mathbf{x}_{i}=\left(x_{i 0}, \ldots, x_{i n_{i}}\right)$. Similarly, for a point $\mathbf{m} \in \mathbb{Z}^{n_{1}+1} \times$ $\cdots \times \mathbb{Z}^{n_{r}+1}$ we set $\mathbf{m}=\left(\mathbf{m}_{1}, \ldots, \mathbf{m}_{r}\right)$ with $\mathbf{m}_{i}=\left(m_{i 0}, \ldots, m_{i n_{i}}\right)$. Define the product $\mathbf{x}^{\mathbf{m}}=\prod_{i, j} x_{i j}^{m_{i j}}$ and the set

$$
M=\left\{\mathbf{m} \in \mathbb{Z}^{n_{1}+1} \times \cdots \times \mathbb{Z}^{n_{r}+1} \mid m_{i j} \geq 0, \sum_{j} m_{i j}=d_{i} \forall i\right\}
$$

so that the polynomial $F$ may be written

$$
F(\mathbf{x})=\sum_{\mathbf{m} \in M} s_{\mathbf{m}} \mathbf{x}^{\mathbf{m}}
$$

where the $s_{\mathbf{m}}$ are totally real algebraic integers. Let $\left\{G_{k}(x)\right\}$ be a finite collection of multihomogeneous polynomials over $K$ with algebraic integer coefficients. Assume that $G_{k}$ has multidegrees $d_{k 1}, \ldots, d_{k r}$. As above, we define the sets

$$
M_{k}=\left\{\mathbf{m} \in \mathbb{Z}^{n_{1}+1} \times \cdots \times \mathbb{Z}^{n_{r}+1} \mid m_{i j} \geq 0, \sum_{j} m_{i j}=d_{k i} \forall i\right\}
$$

and write

$$
G_{k}(\mathbf{x})=\sum_{\mathbf{m} \in M_{k}} s_{k \mathbf{m}} \mathbf{x}^{\mathbf{m}}
$$

where the $s_{k \mathbf{m}}$ are algebraic integers.

If $K$ is a number field containing the coefficients of the polynomials $G_{k}$ and $v$ is a place of $K$ we write $X(K)$ to denote the zero set of $F$ in $\mathcal{P}(K)$ and $X\left(K_{v}\right)$ for the zero set of $F$ in $\mathcal{P}\left(K_{v}\right)$. Let

$$
\begin{aligned}
\Delta_{v}(K) & =\left\{\mathbf{x} \in \mathcal{P}(K) \mid\left\|x_{i j}\right\|_{v} \leq 1 \forall i, j\right\}, \\
\Delta\left(K_{v}\right) & =\left\{\mathbf{x} \in \mathcal{P}\left(K_{v}\right) \mid\left\|x_{i j}\right\|_{v} \leq 1 \forall i, j\right\} .
\end{aligned}
$$

Then define $X_{v}(K)_{1}=X(K) \cap \Delta_{v}(K)$ and $X\left(K_{v}\right)_{1}=X\left(K_{v}\right) \cap \Delta\left(K_{v}\right)$ and observe that $X_{v}(K)_{1} \subset X\left(K_{v}\right)_{1}$. Our first lemma is an analog of Lemma 3.1 of [1].

Lemma 3.1. Suppose that $K$ is any number field containing the coefficients of the polynomials $G_{k}$, that $v$ indexes the places of $K$, and that $a_{k} \geq 0$ for all k. Set

$$
w_{i}=\sum_{k} a_{k} d_{k i}, \quad \log \lambda_{v}=-\max _{\mathbf{x} \in X\left(K_{v}\right)_{1}}\left\{\sum_{k} a_{k} \log \left\|G_{k}(\mathbf{x})\right\|_{v}\right\} .
$$

If $\mathbf{x} \in X(K)$ with $\prod_{k} G_{k}(\mathbf{x}) \neq 0$ then

$$
\sum_{i=1}^{r} w_{i} \log H\left(\mathbf{x}_{i}\right) \geq \sum_{v \mid \infty} \frac{D_{v}}{D} \log \lambda_{v}
$$


Proof. We will prove that the local inequality

$$
\sum_{i=1}^{r} w_{i} \log \left(\max _{j}\left\|x_{i j}\right\|_{v}\right) \geq \sum_{k} a_{k} \log \left\|G_{k}(\mathbf{x})\right\|_{v}+ \begin{cases}\log \lambda_{v} & \text { if } v \mid \infty \\ 0 & \text { if } v \nmid \infty\end{cases}
$$

holds for all places $v$ of $K$.

We first assume that $v \nmid \infty$. Since each coefficient $s_{k \mathbf{m}}$ of $G_{k}$ is an algebraic integer, we have $\left\|s_{k \mathbf{m}}\right\|_{v} \leq 1$ for all $k, \mathbf{m}$. By the strong triangle inequality, there exists $\mathbf{m} \in M$ such that

$$
\begin{aligned}
\sum_{k} a_{k} \log \left\|G_{k}(\mathbf{x})\right\|_{v} & \leq \sum_{k} a_{k} \log \left\|\mathbf{x}^{\mathbf{m}}\right\|_{v}=\sum_{k} a_{k} \sum_{i} d_{k i} \log \max _{j}\left\|x_{i j}\right\|_{v} \\
& =\sum_{i} w_{i} \log \max _{j}\left\|x_{i j}\right\|_{v}
\end{aligned}
$$

and we have established (3.1) in the case that $v \nmid \infty$.

Next we assume that $v \mid \infty$. For each $i$, let $j_{0}=j_{0}(i)$ be such that $\max _{j}\left\|x_{i j}\right\|_{v}=\left\|x_{i j_{0}}\right\|_{v}$. Let $\mathbf{x}^{\prime}$ be the point obtained by replacing each coordinate of $\mathbf{x}$ with $x_{i j} / x_{i j_{0}}$. We have $\left\|x_{i j} / x_{i j_{0}}\right\|_{v} \leq 1$ for all $i, j$ so that

$$
\sum_{i=1}^{r} w_{i} \log \max _{j}\left\|\frac{x_{i j}}{x_{i j_{0}}}\right\|_{v} \geq \sum_{k} a_{k} \log \left\|G_{k}\left(\mathbf{x}^{\prime}\right)\right\|_{v}+\log \lambda_{v} .
$$

By the homogeneity of the polynomials $G_{k}$ we find that

$$
\begin{aligned}
\sum_{k} a_{k} \log \left\|G_{k}\left(\mathbf{x}^{\prime}\right)\right\|_{v} & =\sum_{k} a_{k} \log \left\|\prod_{i} x_{i j_{0}}^{-d_{k i}} G_{k}(\mathbf{x})\right\|_{v} \\
& =\sum_{k} a_{k} \log \left\|G_{k}(\mathbf{x})\right\|_{v}-\sum_{i} w_{i} \log \left\|x_{i j_{0}}\right\|_{v}
\end{aligned}
$$

and conclude that

$$
\sum_{i=1}^{r} w_{i} \log \left(\max _{j}\left\|x_{i j}\right\|_{v}\right) \geq \sum_{k} a_{k} \log \left\|G_{k}(\mathbf{x})\right\|_{v}+\log \lambda_{v}
$$

so we have established (3.1). Now sum both sides of (3.1) over all places $v$ of $K$ and apply the product formula. The desired result follows.

Note that in the version of Lemma 3.1 that appears in [1], the polynomials $G_{k}$ are assumed to have integer coefficients. Therefore, each $\lambda_{v}$ is in fact independent of $v$. In this simpler situation, Beukers and Zagier define $\lambda_{v}$ using the usual absolute value on $\mathbb{C}$ rather than $\|\cdot\|_{v}$ on $K_{v}$.

In our version of Lemma 3.1 we allow for the $G_{k}$ to have any algebraic integer coefficients, so we must define $\lambda_{v}$ using $\|\cdot\|_{v}$ on a number field containing the coefficients of the $G_{k}$. It is certainly possible that $\lambda_{v}$ does indeed depend on the place $v$. However, with appropriate choices for $G_{k}$ and $a_{k}$, conditions (i) and (ii) are enough to produce a universal lower bound 
on $\lambda_{v}$ that does not depend on $v$. In view of Lemma 3.1, this lower bound gives a bound on $\sum_{i=1}^{r}\left(n_{i}+1\right) \log H\left(\mathbf{x}_{i}\right)$.

Before we make selections for the $G_{k}$ and the $a_{k}$, we state Lemmas 3.2 and 3.3 of [1] for later use. Although the statement of Lemma 3.2 in [1] is for polynomials with integer coefficients, it is easily verified that the lemma holds for polynomials with complex coefficients and we state this generalization here.

Lemma 3.2. Suppose $v$ is an Archimedean place of a number field $K$ with $D_{v}=2$. If $Q_{k}(\mathbf{x})$ are multihomogeneous polynomials with coefficients in $K_{v}$ then the function $\sum_{k} a_{k} \log \left\|Q_{k}(\mathbf{x})\right\|_{v}$ assumes a maximum in $X\left(K_{v}\right)_{1}$ at a point $\mathbf{x}$. Moreover, $\mathbf{x}$ has one coordinate pair $\left(i_{0}, j_{0}\right)$ such that $\left\|x_{i j}\right\|_{v}=1$ for all $(i, j) \neq\left(i_{0}, j_{0}\right)$.

Lemma 3.3. Let $\alpha, \beta, \gamma>0$. Let $l$ be the unique minimum of the function

$$
u \log \frac{\gamma u}{u+v}+v \log \frac{v}{u+v}
$$

under the constraints $u, v \geq 0, \alpha u+\beta v=1$. Then $e^{-l}$ is the unique real root larger than 1 of $\gamma^{-1} x^{-\alpha}+x^{-\beta}=1$.

We now make our selections for $G_{k}$ and $a_{k}$ following [1]. For $G_{k}$ we choose the coordinates $x_{i j}$ and the polynomial

$$
\widetilde{F}(\mathbf{x})=F\left(\mathbf{x}^{-1}\right) \prod_{i, j} x_{i j}^{d_{i j}}
$$

Note that $\widetilde{F}$ is multihomogeneous with multidegrees given by $\widetilde{d}_{i}=-\widetilde{d}_{i}$ $+\sum_{j} d_{i j}$. Write $a_{i j}$ and $b$ for the values of $a_{k}$ corresponding to $x_{i j}$ and $\widetilde{F}$, respectively. In this situation we have

$$
\log \lambda_{v}=-\max _{\mathbf{x} \in X\left(K_{v}\right)_{1}}\left\{\sum_{i, j} a_{i j} \log \left\|x_{i j}\right\|_{v}+b \log \|\widetilde{F}(\mathbf{x})\|_{v}\right\} .
$$

Finally, let $\varrho$ be the real root greater than 1 of $x^{-2}+C_{F}^{-1} x^{-\delta}=1$.

Lemma 3.4. Suppose $K$ is a number field containing the coefficients of $F$ and $D_{v}=2$ for all Archimedean places $v$ of $K$. Then there exist $a_{i j}, b \geq 0$ such that $n_{i}+1=\sum_{j} a_{i j}+b \widetilde{d}_{i}$ for all $i$ and $\lambda_{v} \geq \varrho$ for all $v \mid \infty$.

Proof. Following [1], we define each $a_{i j}$ in terms of $b$ by

$$
a_{i j}=1-\frac{\widetilde{d}_{i}}{n_{i}+1} b \quad \text { if } i \notin I
$$

and

$$
a_{i j}=1-\frac{\widetilde{d}_{i}+(-1)^{j} d_{i 1}}{n_{i}+1} b \quad \text { if } i \in I
$$


so we immediately have $n_{i}+1=\sum_{j} a_{i j}+b \widetilde{d}_{i}$. Now we need only select $b$ so that $\lambda_{v} \geq \varrho$.

We will show that under the assumptions (3.3) and (3.4),

$$
-\log \lambda_{v} \leq b \log \frac{2 b C_{F}}{(1-\delta b)+2 b}+\frac{1-\delta b}{2} \log \frac{1-\delta b}{(1-\delta b)+2 b}
$$

for every Archimedean place $v$ of $K$. Let

$$
\Phi(\mathbf{x})=\sum_{i, j} a_{i j} \log \left\|x_{i j}\right\|_{v}+b \log \|\widetilde{F}(\mathbf{x})\|_{v}
$$

so that we must give an upper bound on $-\log \lambda_{v}=\max _{\mathbf{x} \in X\left(K_{v}\right)_{1}} \Phi(\mathbf{x})$. By Lemma 3.2 this maximum is attained at a point $\mathbf{x} \in X\left(K_{v}\right)_{1}$ where $\left\|x_{i_{0} j_{0}}\right\|_{v} \leq 1$ for some coordinate pair $\left(i_{0}, j_{0}\right)$ and $\left\|x_{i j}\right\|_{v}=1$ for all $(i, j)$ $\neq\left(i_{0}, j_{0}\right)$. Hence, $\bar{x}_{i j}=x_{i j}^{-1}$ for all $(i, j) \neq\left(i_{0}, j_{0}\right)$. Moreover, $\Phi(\mathbf{x}) \rightarrow-\infty$ as $x_{i j} \rightarrow 0$ for any $i, j$. Therefore, we must have $x_{i_{0} j_{0}} \neq 0$ so that the point $\mathbf{x}^{-1}$ is well defined.

Suppose first that $\left(i_{0}, j_{0}\right) \notin E$ and write $x=x_{i_{0} j_{0}}, d=d_{i_{0} j_{0}}$ and $m=m_{i_{0} j_{0}}$ for any $\mathbf{m} \in M$. Let $\overline{\mathbf{x}}$ be the point obtained by replacing each coordinate of $\mathbf{x}$ with $\bar{x}_{i j}$. By property (i), the coefficients of $F$ are in the fixed field of complex conjugation in $K_{v}$. Using $F(\mathbf{x})=0$ we obtain

$$
\begin{aligned}
F\left(\mathbf{x}^{-1}\right) & =F\left(\mathbf{x}^{-1}\right)-F(\overline{\mathbf{x}})=\sum_{\mathbf{m} \in M} s_{\mathbf{m}} \mathbf{x}^{-\mathbf{m}}-\sum_{\mathbf{m} \in M} s_{\mathbf{m}} \overline{\mathbf{x}}^{\mathbf{m}} \\
& =\sum_{\mathbf{m} \in M} s_{\mathbf{m}}\left(\frac{\overline{\mathbf{x}}^{\mathbf{m}}}{\bar{x}^{m}}\right)\left(x^{-m}-\bar{x}^{m}\right)
\end{aligned}
$$

and note that $\left\|\overline{\mathbf{x}}^{\mathbf{m}} / \bar{x}^{m}\right\|_{v}=1$ for all $\mathbf{m} \in M$. We now apply the triangle inequality to find

$$
\begin{aligned}
\|\widetilde{F}(\mathbf{x})\|_{v} & \leq\|x\|_{v}^{d} \sum_{\mathbf{m} \in M}\left\|s_{\mathbf{m}}\left(x^{-m}-\bar{x}^{m}\right)\right\|_{v} \\
& \leq\|x\|_{v}^{d}\left\|x^{-1}-\bar{x}\right\|_{v} \sum_{\mathbf{m} \in M} m\left\|s_{\mathbf{m}}\right\|_{v}\left\|x^{-1}\right\|_{v}^{m-1} \\
& \leq\|x\|_{v}^{d}\left\|x^{-1}-\bar{x}\right\|_{v}\|x\|_{v}^{1-d} \sum_{\mathbf{m} \in M} m\left\|s_{\mathbf{m}}\right\|_{v}=c\left(F, v, i_{0}, j_{0}\right)\left(1-\|x\|_{v}^{2}\right),
\end{aligned}
$$

where the last equality follows since $c\left(F, v, i_{0}, j_{0}\right)=\sum_{\mathbf{m} \in M} m\left\|s_{\mathbf{m}}\right\|_{v}$.

Now let $\xi=\|x\|_{v}^{2}, c=c\left(F, v, i_{0}, j_{0}\right)$ and $a=a_{i_{0} j_{0}}$. We have

$$
-\log \lambda_{v} \leq \max _{\xi \in[0,1]}\left(b \log (c(1-\xi))+\frac{a}{2} \log \xi\right) .
$$

Differentiating we find that this maximum is attained at $\xi_{0}=a /(a+2 b)$ and its value is

$$
b \log \frac{2 b c}{a+2 b}+\frac{a}{2} \log \frac{a}{a+2 b} .
$$


By definition $a=1-b \widetilde{d}_{i} /\left(n_{i}+1\right) \geq 1-\delta b$. Therefore (3.6) is bounded above by

$$
b \log \frac{2 b C_{F}}{(1-\delta b)+2 b}+\frac{1-\delta b}{2} \log \frac{1-\delta b}{(1-\delta b)+2 b}
$$

and (3.5) follows.

Next assume that $\left(i_{0}, j_{0}\right) \in E$ so that $j_{0}=0$. We have $\left\|x_{i_{0} 0}\right\| \leq 1$ and $\left\|x_{i j}\right\|=1$ for all $(i, j) \neq\left(i_{0}, 0\right)$. We write $x=x_{i_{0} 0}, x^{\prime}=x_{i_{0} 1}, d=d_{i_{0} 0}$, $d^{\prime}=d_{i_{0} 1}, m=m_{i_{0} 0}$ and $m^{\prime}=m_{i_{0} 1}$ for each $\mathbf{m} \in M$. Then we find

$$
\begin{aligned}
\|\widetilde{F}(\mathbf{x})\|_{v} & =\left\|x^{d} F\left(\mathbf{x}^{-1}\right)-\bar{x}^{-d} F(\overline{\mathbf{x}})\right\|_{v}=\left\|\sum_{\mathbf{m} \in M} s_{\mathbf{m}}\left(\frac{\overline{\mathbf{x}}^{\mathbf{m}}}{\bar{x}^{m}}\right)\left(x^{d-m}-\bar{x}^{m-d}\right)\right\|_{v} \\
& \leq \sum_{\mathbf{m} \in M}\left\|s_{\mathbf{m}}\left(x^{d-m}-\bar{x}^{m-d}\right)\right\|_{v} \\
& \leq\left\|x-\bar{x}^{-1}\right\|_{v} \sum_{\mathbf{m} \in M}(d-m)\left\|s_{\mathbf{m}}\right\|_{v}\left\|x^{-1}\right\|_{v}^{d-m-1} .
\end{aligned}
$$

We know that $m+m^{\prime}=d_{i_{0}} \geq d$ so $d-m \leq m^{\prime}$. Therefore,

$$
\begin{aligned}
\|\widetilde{F}(\mathbf{x})\|_{v} & \leq\left\|x-\bar{x}^{-1}\right\|_{v} \sum_{\mathbf{m} \in M} m^{\prime}\left\|s_{\mathbf{m}}\right\|_{v}\|x\|_{v}^{1-m^{\prime}} \\
& \leq\left\|x-\bar{x}^{-1}\right\|_{v}\|x\|_{v}^{1-d^{\prime}} \sum_{\mathbf{m} \in M} m^{\prime}\left\|s_{m}\right\|_{v}=\left(1-\|x\|_{v}^{2}\right)\|x\|_{v}^{-d^{\prime}} c\left(F, v, i_{0}, 1\right) .
\end{aligned}
$$

Let $\xi=\|x\|_{v}^{2}$ and $c=c\left(F, v, i_{0}, 1\right)$ so that

$$
\log \lambda_{v} \leq \max _{\xi \in[0,1]}\left(b \log (c(1-\xi))-\frac{d_{i_{0} 1} b}{2} \log \xi+\frac{a_{i_{0} 0}}{2} \log \xi\right) .
$$

With $a=a_{i_{0} 0}-d^{\prime} b$ we observe that the right hand side of (3.7) equals

$$
b \log \frac{2 b c}{a+2 b}+\frac{a}{2} \log \frac{a}{a+2 b} .
$$

It follows from (3.4) that $a \geq 1-\delta b$ and (3.5) holds.

Finally, we select $b$ to make the right hand side of (3.5), which does not depend on $v$, as small as possible. Then we make choices for $a_{i j}$ according to (3.3) and (3.4). We apply Lemma 3.3 with $\alpha=\delta, \beta=2, \gamma=C_{F}, u=b$ and $v=(1-\delta b) / 2$. By the lemma, the right hand side of (3.5) has a unique minimum $l$ where $e^{-l}$ is the unique real root larger than 1 of $x^{-2}+C_{F} x^{-\delta}=1$. Setting $\varrho=e^{-l}$ we establish the lemma.

Proof of Theorem 2.1. Suppose $\mathbf{x} \in \mathcal{P}(\overline{\mathbb{Q}})$ and $K$ is a number field containing all coordinates of $\mathbf{x}$ and all coefficients of $F$ and having $D_{v}=2$ for all $v \mid \infty$. Assume $a_{i j}, b$ are the constants from Lemma 3.4 and $\lambda_{v}$ is defined as in (3.2). Since $x_{i j}$ and $\widetilde{F}$ are multihomogeneous and $n_{i}+1=\sum_{j} a_{i j}+b \widetilde{d}_{i}$, 
Lemma 3.1 implies that

$$
\sum_{i=1}^{r}\left(n_{i}+1\right) \log H\left(\mathbf{x}_{i}\right) \geq \sum_{v \mid \infty} \frac{d_{v}}{d} \log \lambda_{v}
$$

whenever $x_{i j} \neq 0$ for all $i, j$ and $F\left(\mathbf{x}^{-1}\right) \neq 0$. Then by Lemma 3.4 we have $\lambda_{v} \geq \varrho$ so that

$$
\sum_{i=1}^{r}\left(n_{i}+1\right) \log H\left(\mathbf{x}_{i}\right) \geq \sum_{v \mid \infty} \frac{d_{v}}{d} \log \varrho=\log \varrho .
$$

Acknowledgments. The author wishes to thank Professor J. D. Vaaler for many useful discussions regarding this work.

\section{References}

[1] F. Beukers and D. Zagier, Lower bounds of heights of points on hypersurfaces, Acta Arith. 79 (1997), 103-111.

[2] E. Dobrowolski, On a question of Lehmer and the number of irreducible factors of a polynomial, ibid. 34 (1979), 391-401.

[3] D. H. Lehmer, Factorization of certain cyclotomic functions, Ann. of Math. 34 (1933), 461-479.

[4] A. Schinzel, On the product of the conjugates outside the unit circle of an algebraic number, Acta Arith. 24 (1973), 385-399; Addendum, ibid. 26 (1975), 329-331.

[5] C. J. Smyth, On the product of the conjugates outside the unit circle of an algebraic integer, Bull. London Math. Soc. 3 (1971), 169-175.

[6] D. Zagier, Algebraic numbers close to both 0 and 1, Math. Comp. 61 (1993), 485-491.

[7] S. Zhang, Positive line bundles on arithmetic surfaces, Ann. of Math. 136 (1992), $569-587$.

Department of Mathematics

University of Texas at Austin

1 University Station C1200

Austin, TX 78712, U.S.A.

E-mail: csamuels@math.utexas.edu

Received on 22.11.2005

and in revised form on 13.4.2006 